Efthimiopoulos, I., Mayanna, S., Stavrou, E., Torode, A., Wang, Y. (2020): Extracting the Anharmonic Properties of the G-Band in Graphene Nanoplatelets. - Journal of Physical Chemistry C, 124, 8, 4835-4842.

https://doi.org/10.1021/acs.jpcc.9b10875 


\title{
Extracting the Anharmonic Properties of the G-band in Graphene Nanoplatelets
}

\author{
Ilias Efthimiopoulos ${ }^{a, b, *}$, Sathish Mayanna ${ }^{c, d}$, Elissaios Stavrou ${ }^{e}$, Antonius Torode ${ }^{a}$, and Yuejian \\ Wang $^{a, *}$ \\ ${ }^{a}$ Department of Physics, Oakland University, Rochester, Michigan 48309, USA \\ bDeutsches GeoForschungsZentrum, Section 3.6, Telegrafenberg, 14473, Potsdam, Germany \\ 'Deutsches GeoForschungsZentrum, Section 3.5, Telegrafenberg, 14473, Potsdam, Germany \\ ${ }^{\mathrm{d} C a r l}$ Zeiss AG, Carl-Zeiss-Strasse 22, 73447, Oberkochen, Germany \\ étawrence Livermore National Laboratory, Physical and Life Sciences Directorate, P.O. Box 808, L-350, Livermore, \\ California 94550, USA
}

\begin{abstract}
We have examined the effect of temperature and pressure on the Raman-active G-band of graphene nanoplatelets with an average thickness of $4 \mathrm{~nm}$. Measuring the Raman mode frequency and width of the G-band as a function of temperature and pressure allowed us to extract the individual factors contributing to the frequency and width of the G-band, i.e. the volumetric (thermal expansion) and anharmonic (phonon-phonon interactions) terms responsible for the Raman shift, and the electron-phonon coupling and anharmonic contributions controlling the width/ lifetime of the G-band. Considering the available literature, the significant role of the anharmonic effects for understanding the G-band physical processes in carbon-based systems is highlighted.
\end{abstract}

\footnotetext{
*Corresponding authors: ilias.efthymiopoulos@gfz-potsdam.de and ywang235@oakland.edu
} 


\section{INTRODUCTION}

Raman spectroscopic investigations under external perturbations constitute an indispensible tool for understanding partially the physical phenomena associated with anharmonic phonon processes, such as thermal expansion, specific heat, and thermal conductivity ${ }^{1-6}$. These anharmonic phonon effects encompass phonon-phonon and electron-phonon interactions, which play in turn a key role in the thermal and transport properties of materials.

In graphene and relevant carbon (nano)materials, in particular, monitoring of the intense Raman-active G-phonon band with varying temperatures has become a routine for extracting information regarding the thermal transport properties of these systems ${ }^{7-10}$. These experiments allow for quantifiying important physical properties such as thermal conductivity ${ }^{11-13}$, which in turn constitute key ingredients for the design of efficient graphene-based nanoelectronic devices ${ }^{14}$. There exists, however, a scatter in the obtained Raman-related temperature coefficients, arising in part from the presence of supporting substrates under the graphene layers, which affect the intrinsic response of graphene due to e.g. the size mismatch (strain effects) induced by the different thermal expansion behavior ${ }^{3,15,16}$. Consequently, unraveling the intrinsic anharmonic phonon properties of free-standing graphene samples becomes a necessary and imperative task, in order to acquire the 'real' temperature effects in these important materials.

Here we have conducted temperature-dependent Raman scattering experiments on graphene nanoplatelets (GNPs) with an average thickness of less than $4 \mathrm{~nm}$, corresponding to almost 12 graphene layers stacked on top of each other. As GNPs do not require any supporting substrate to facilitate experimental measurements, and can be viewed as an 'intermediate' between graphene and bulk graphite from a structural perspective ${ }^{17}$, GNPs offer the advantages of (a) extracting the intrinsic temperature response of the graphene layers and (b) serve as a 'bridge' between threedimensional graphite and two-dimensional graphene regarding the understanding of the anharmonic phonon processes. Moreover, by performing also high-pressure Raman investigations, we were able to model the temperature dependence of the Raman shift and width of the GNPs' G-band ${ }^{1,4,6,18,19}$. Comparison between our results and literature data ${ }^{1,20-23}$ clearly reveals the important role of phonon-phonon interactions in controlling the thermal transport properties of these materials. 


\section{EXPERIMENTAL METHODS}

The graphene nanoplatelets with an average thickness of less than $4 \mathrm{~nm}$, corresponding to approximately 12 graphene layers, were purchased commercially. The samples have been characterized by Field emission Scanning electron microscopy (FE-SEM), energy-dispersive Xray spectroscopy (EDS), Raman spectroscopy, and X-ray diffraction (XRD) at ambient conditions.

The temperature-dependent Raman measurements at ambient pressure were conducted with a single-stage Raman spectrometer, coupled to a Charged-Coupled Device (CCD) and a green solid-state laser $(\lambda=532 \mathrm{~nm})$. The laser beam was focused on the sample surface with a $20 \times$ objective lens, an incident laser power of $\sim 1 \mathrm{~mW}$, whereas the collection time of each spectrum was set to 3 accumulations of 60 seconds each. The spectrometer resolution with this configuration is estimated at $1 \mathrm{~cm}^{-1}$, considering the Rayleigh and laser plasma lines. The sample was placed inside a Linkam THMS600 stage, purged continuously with nitrogen gas. The thermocouple readout temperature has been corrected with an empirical formula, derived from the melting points of different salts and the freezing point of water ${ }^{24,25}$.

The high-pressure Raman measurements at ambient temperature were conducted with the same Raman system. Pressure was generated with gasketed diamond anvil cells (DACs) equipped with low-fluorescence type II diamonds of $400 \mu \mathrm{m}$ culet diameters. Rhenium gaskets were preindented to a $30 \mu \mathrm{m}$ thickness, with holes of $150-200 \mu \mathrm{m}$ diameters acting as sample chambers. The incident laser power measured outside the DAC was $\sim 3 \mathrm{~mW}$, whereas the collection time of each Raman spectrum in this series of experiments case was set to 5 accumulations of 120 seconds each. Helium served as a pressure transmitting medium (PTM), whereas the ruby fluorescence method was employed for pressure calibration ${ }^{26}$.

\section{RESULTS AND DISCUSSION}

\section{A. Characterization of the graphene nanoplatelets}

In Fig. 1 we show the SEM images and EDS analysis of the commercially purchased GNPs. The results show that GNPs are in different shapes and sizes having a plate-like morphology. The 
EDS analysis shows that these bulk GNPs samples are not totally pure however, contains impurities which are specifically rich in Fe and Cr.

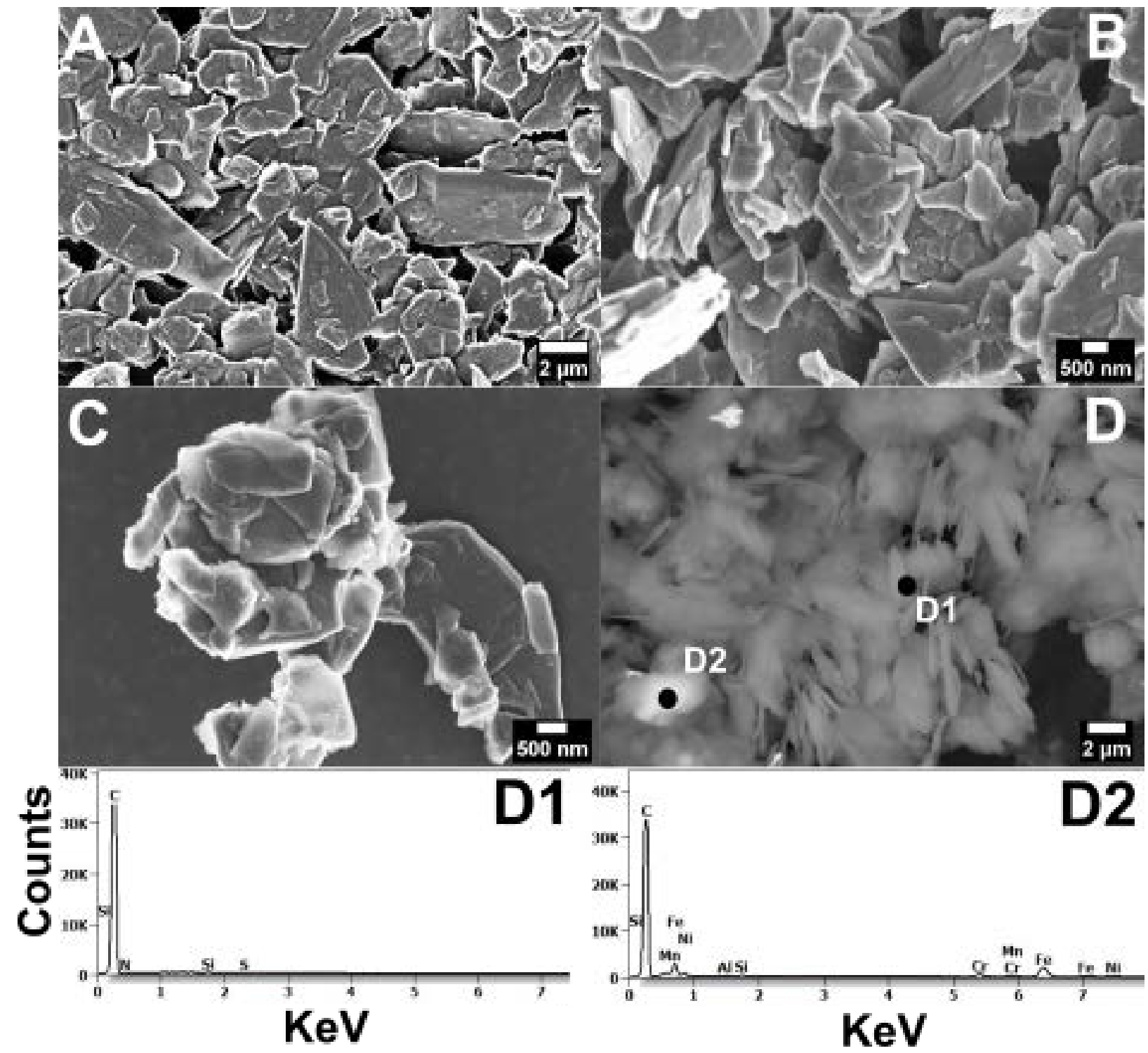

Figure 1. (A-C) Secondary electron images of the commercial graphene nanoplatelet samples, which exhibit varying particle shapes sizes and shapes. (D) Backscattered electron image showing the (D2) presence of few contaminants which are typically Fe- and Cr-rich nanoparticles. 


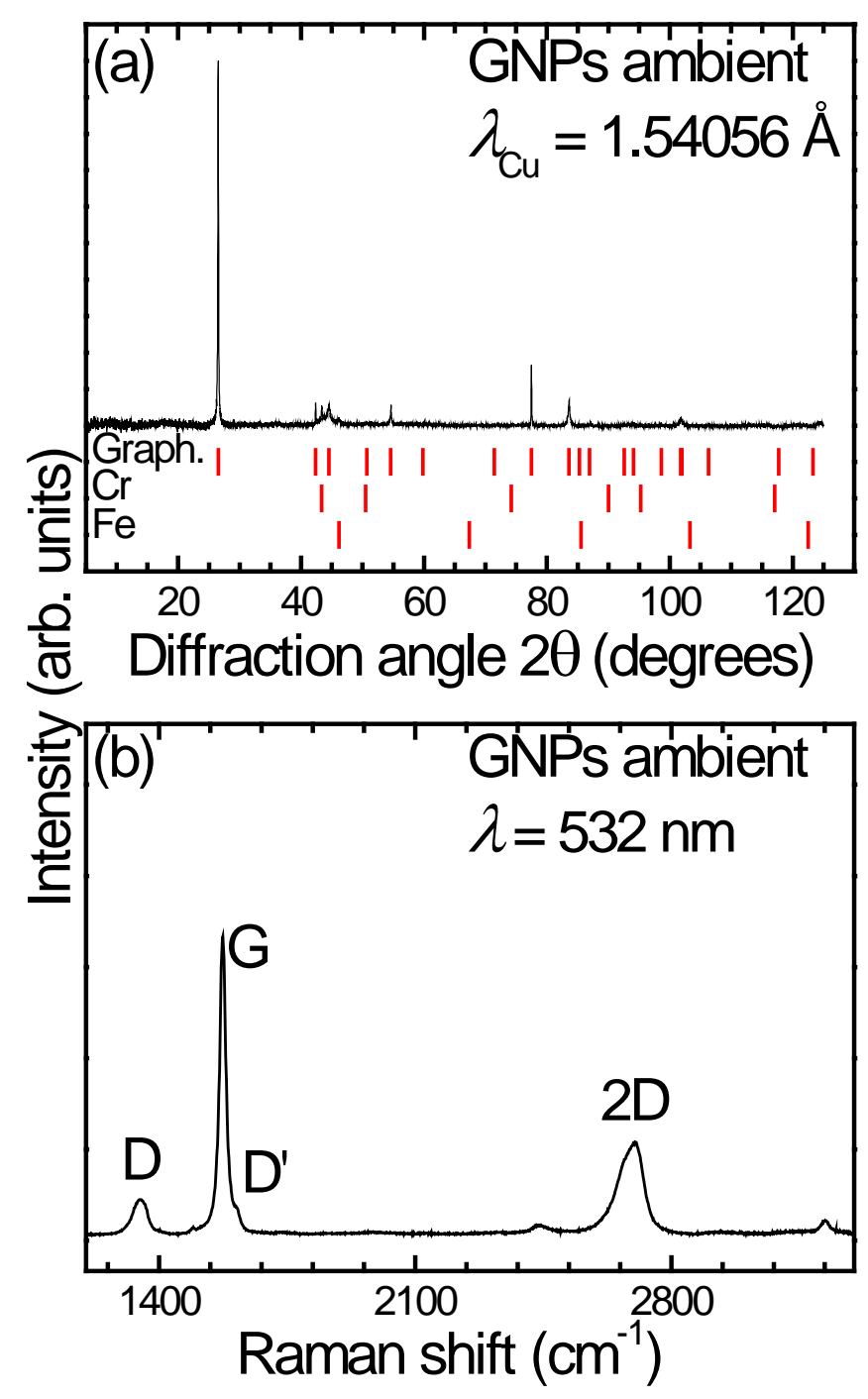

Figure 2. (a) XRD pattern and (b) Raman spectrum of the synthetic GNPs at ambient conditions.

In Fig. 2 we show the XRD pattern and Raman spectrum of the investigated GNPs at ambient conditions. Even though the graphite crystalline phase is the dominant one, we can observe the additional Bragg peaks arising from the metal impurities in the synthetic sample. With the help of the EDS results, we can assign these metal impurities to elemental $\mathrm{Cr}$ and $\mathrm{Fe}^{27}$. As for the dominant graphitic phase, the extracted structural parameters are: $a=2.4616(2) \AA, c=6.719(1)$ $\AA$, the volume $V=35.26 \AA^{3}$, and the axial ratio $c / a=2.73$, very close to the respective structural parameters of bulk graphite ${ }^{28}$.

On the other hand, the Raman spectrum of the GNPs comprises of three major (and one minor) bands at ambient conditions, consistent with the general consensus for graphitic systems ${ }^{29-31}$ : the 
most intense Raman feature at $1580 \mathrm{~cm}^{-1}$ corresponds to the G-band, a doubly degenerate Ramanactive vibration ( $E_{2 g}$ symmetry) assigned to the stretching motions of $\mathrm{sp}^{2}$-bonded carbon ions, whereas the three remaining Raman features at $\sim 1350 \mathrm{~cm}^{-1}$ (D-band), its overtone at $2700 \mathrm{~cm}^{-1}$ (2D-band), and the low-lying Raman feature at $1620 \mathrm{~cm}^{-1}\left(\mathrm{D}^{\prime}\right)$ originate from defects and/or disorder. Finally, the relatively weak intensity of the D-band compared to the G-band in the recorded GNPs Raman spectra indicates the good quality of the investigated samples ${ }^{29-32}$, consistent with our SEM and XRD observations.

\section{B. Temperature dependence of the G-band in GNPs}

In Fig. 3(a) we plot the measured Raman spectra of GNPs at various temperatures. We should point out here that since we were not able to detect the $\mathrm{D}$ and 2D features in our high-pressure Raman experiments due to the diamond anvils' intense and overlapping Raman signals, we chose to investigate the effect of temperature and pressure on the GNPs' G-band alone. We can observe that upon increasing temperature, the frequency of the G-band shifts to lower wavenumbers [Fig. 3(b)], whereas its full width at half maximum (FWHM) displays a decrease (within error) in the investigated 100-700 K temperature range [Fig. 3(c)]. 

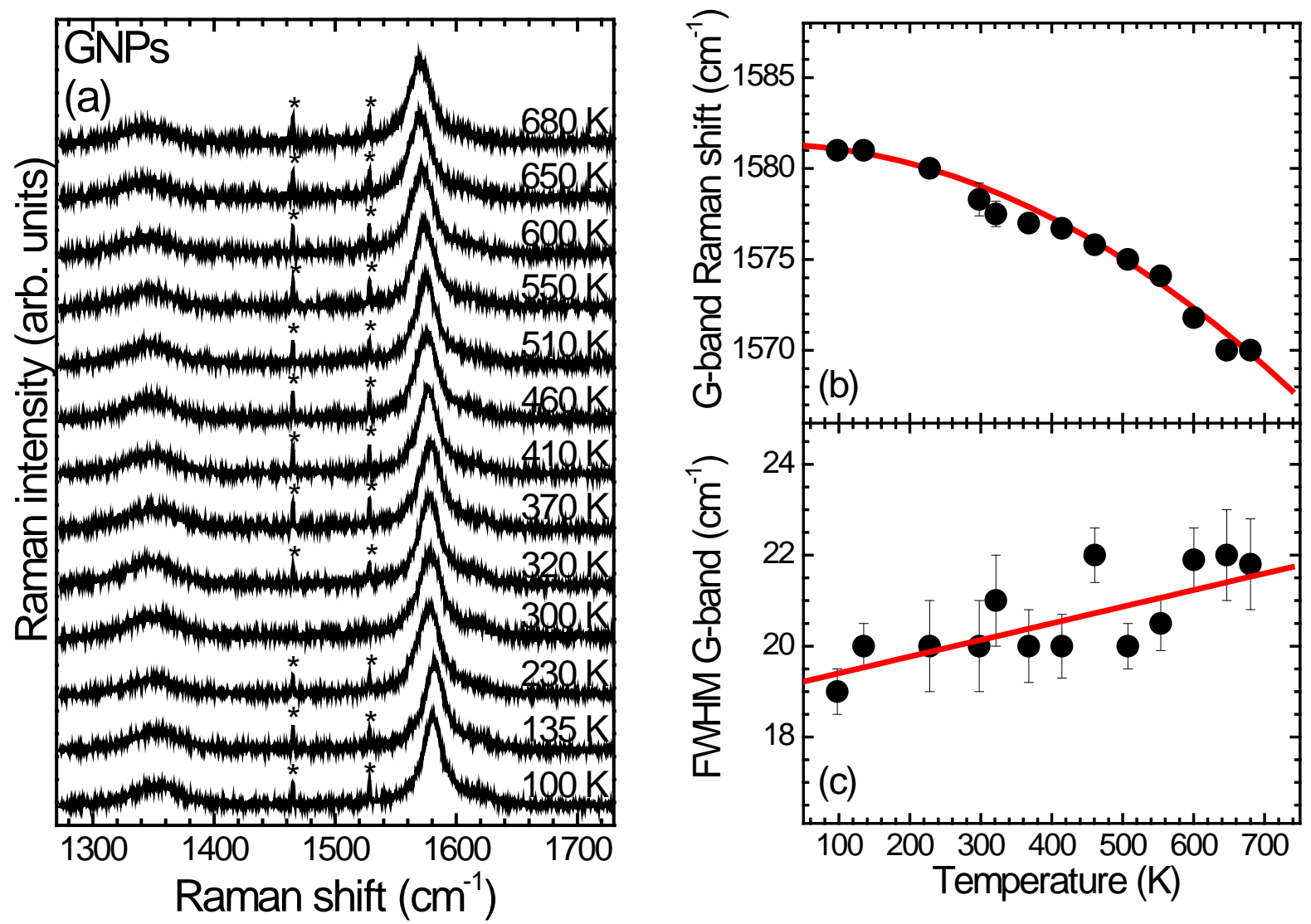

Figure 3. (a) Selected Raman spectra of GNPs at various temperatures within the $1270-1720 \mathrm{~cm}^{-1}$ frequency region of interest $(\lambda=532 \mathrm{~nm}, P=1 \mathrm{bar})$. Asterisks mark laser plasma lines. The spectra have been scaled with respect to the intense G-band. Dependence of the (b) Raman mode frequency and (c) full width at half maximum (FWHM) of the G-band against temperature. The spectrometer width contribution of $1 \mathrm{~cm}^{-1}$ has been substracted from the FWHM data. Solid lines passing through the measured data correspond to polynomial fittings.

The isobaric Raman mode frequency evolution of the G-band as a function of temperature can be fitted to the following polynomial expression:

$$
\omega_{i}^{P}(T)=\omega_{i 0}^{P}+\left(\frac{\partial \omega_{i}}{\partial T}\right)_{P} \Delta T+\left(\frac{\partial^{2} \omega_{i}}{\partial T^{2}}\right)_{P} \Delta T^{2}
$$

where $\omega_{\mathrm{i} 0}{ }^{P}$ the (extrapolated) mode frequency at zero temperature and ambient pressure, $\Delta T$ the temperature difference, $\left(\partial \omega_{\mathrm{i}} / \partial T\right)_{P}$ and $\left(\partial^{2} \omega_{\mathrm{i}} / \partial T^{2}\right)_{P}$ the first- and second-order temperature coefficient. The respective fitting yields: $\omega_{\mathrm{i} 0}^{P}=1581.4(2) \mathrm{cm}^{-1},\left(\partial \omega_{\mathrm{i}} / \partial T\right)_{P}=-4.8(1) \cdot 10^{-4} \mathrm{~cm}^{-1} / \mathrm{K}$, and $\left(\partial^{2} \omega_{\mathrm{i}} / \partial T^{2}\right)_{P}=-2.4(3) \cdot 10^{-5} \mathrm{~cm}^{-1} / \mathrm{K}^{2}$ [Fig. 3(b) \& Table 1]. 
Table 1. Raman-related temperature coefficients for the G-band of various pristine carbon-based materials. N/A: Not Available.

\begin{tabular}{|c|c|c|c|c|c|c|}
\hline $\begin{array}{l}\text { Carbon samples } \\
\text { (pristine) }\end{array}$ & $\begin{array}{c}\omega_{\mathrm{i} 0}^{P} \\
\left(\mathrm{~cm}^{-1}\right)\end{array}$ & $\begin{array}{l}(\partial \omega / \partial T)_{P} \\
\left(\mathrm{~cm}^{-1} / \mathrm{K}\right)\end{array}$ & $\begin{array}{c}\left(\partial^{2} \omega / \partial T^{2}\right)_{P} \\
\left(\mathrm{~cm}^{-1} / \mathrm{K}^{2}\right)\end{array}$ & $\begin{array}{c}\Gamma_{\mathrm{i} 0}^{P} \\
\left(\mathrm{~cm}^{-1}\right)\end{array}$ & $\begin{array}{l}(\partial \Gamma / \partial T)_{P} \\
\left(\mathrm{~cm}^{-1} / \mathrm{K}\right)\end{array}$ & $\begin{array}{c}\text { Temperature } \\
\text { Range }\end{array}$ \\
\hline GNPs & $1581.4(2)$ & $-4.8(1) \cdot 10^{-4}$ & $-2.4(3) \cdot 10^{-5}$ & $19.0(5)$ & $0.004(1)$ & $100-680 \mathrm{~K}$ \\
\hline Graphite $^{33}$ & N/A & -0.011 & N/A & 12 & N/A & $286-647 \mathrm{~K}$ \\
\hline Graphite $^{23}$ & 1583.5 & $-0.013^{\mathbf{a}}$ & N/A & 12 & N/A & 4-1000 K \\
\hline Graphene (single-layer) $^{3}$ & 1584 & -0.016 & N/A & N/A & N/A & 83-373 K \\
\hline Graphene (single-layer) ${ }^{15}$ & N/A & -0.016 & N/A & N/A & $\sim 0$ & 77-573 K \\
\hline Graphene (bilayer) $^{3}$ & 1582 & -0.015 & N/A & $\mathrm{N} / \mathrm{A}$ & N/A & $113-373 \mathrm{~K}$ \\
\hline Graphene (six-layer) $^{15}$ & N/A & -0.014 & N/A & N/A & -0.022 & 77-573 K \\
\hline Graphene (multilayer) $^{34}$ & $\sim 1586$ & -0.015 & N/A & N/A & N/A & $78-573 \mathrm{~K}$ \\
\hline Graphene sheets $^{22}$ & 1582 & -0.020 & N/A & $\sim 17.5$ & N/A & 79-773 K \\
\hline Diamond $^{35}$ & 1333.5 & -0.012 & N/A & 0.416 & 0.004 & $300-1900 \mathrm{~K}$ \\
\hline
\end{tabular}

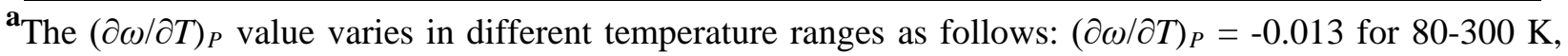
$(\partial \omega / \partial T)_{P}=-0.017$ for $300-500 \mathrm{~K},(\partial \omega / \partial T)_{P}=-0.020$ for $500-700 \mathrm{~K}$, and $(\partial \omega / \partial T)_{P}=-0.026$ for $700-900$ $\mathrm{K}$.

In a similar fashion, the width of the G-band against temperature can be fitted also with a polynomial function [Fig. 3(c)]:

$$
\Gamma_{i}(T)=\Gamma_{i 0}^{P}+\left(\frac{\partial \Gamma_{i}}{\partial T}\right)_{P} \Delta T
$$

with $\Gamma_{\mathrm{i} 0}{ }^{\mathrm{P}}$ the (extrapolated) width at zero temperature and ambient pressure, $\Delta T$ the temperature difference, and $\left(\partial \Gamma_{\mathrm{i}} / \partial T\right)_{P}$ the first-order temperature coefficients. The respective fitting yields: $\Gamma_{\mathrm{i} 0}{ }^{\mathrm{P}}=19.0(5) \mathrm{cm}^{-1}$ and $\left(\partial \Gamma_{\mathrm{i}} / \partial T\right)_{P}=0.004(1) \mathrm{cm}^{-1} / \mathrm{K}$. Our results are tabulated alongside literature data on graphite and graphene samples in Table 1. An interesting point here is that the width of the G-band exhibits a (moderate) broadening upon increasing temperature within the 100-700 K temperature range, unlike bulk graphite ${ }^{1,21,23}$, yet in line with experimental measurements on graphene nanosheets ${ }^{22}$ and theoretical predictions on single-layer graphene $\mathrm{e}^{20}$. According to the available models ${ }^{1,20}$, the temperature dependence of the G-band width is controlled by electron-phonon and phonon-phonon scattering processes. The two terms exhibit opposite behavior with temperature, i.e. the electron-phonon scattering term decreases and the phonon-phonon interaction term increases with increasing temperature. Hence, the GNPs' Gband width broadening observed here indicates the prevailance of the (anharmonic) phonon- 
phonon interactions in the G-band width with increasing temperature. We will return to this point later below.

\section{Raman spectra of G-band under pressure}

Regarding the effect of pressure on the G-band of GNPs, in Fig. 4(a) we show selected Raman spectra of the graphene nanoplatelets in the vicinity of the G-band at various pressures up to $\sim 10$ GPa. The G-band mode frequency displays a normal behavior upon compression, i.e. it exhibits a positive pressure dependence [Fig. 4(b)], whereas its FWHM is almost insensitive within the examined pressure range, consistent with previous results on GNPs ${ }^{36}$ and bulk graphite ${ }^{37,38}$.
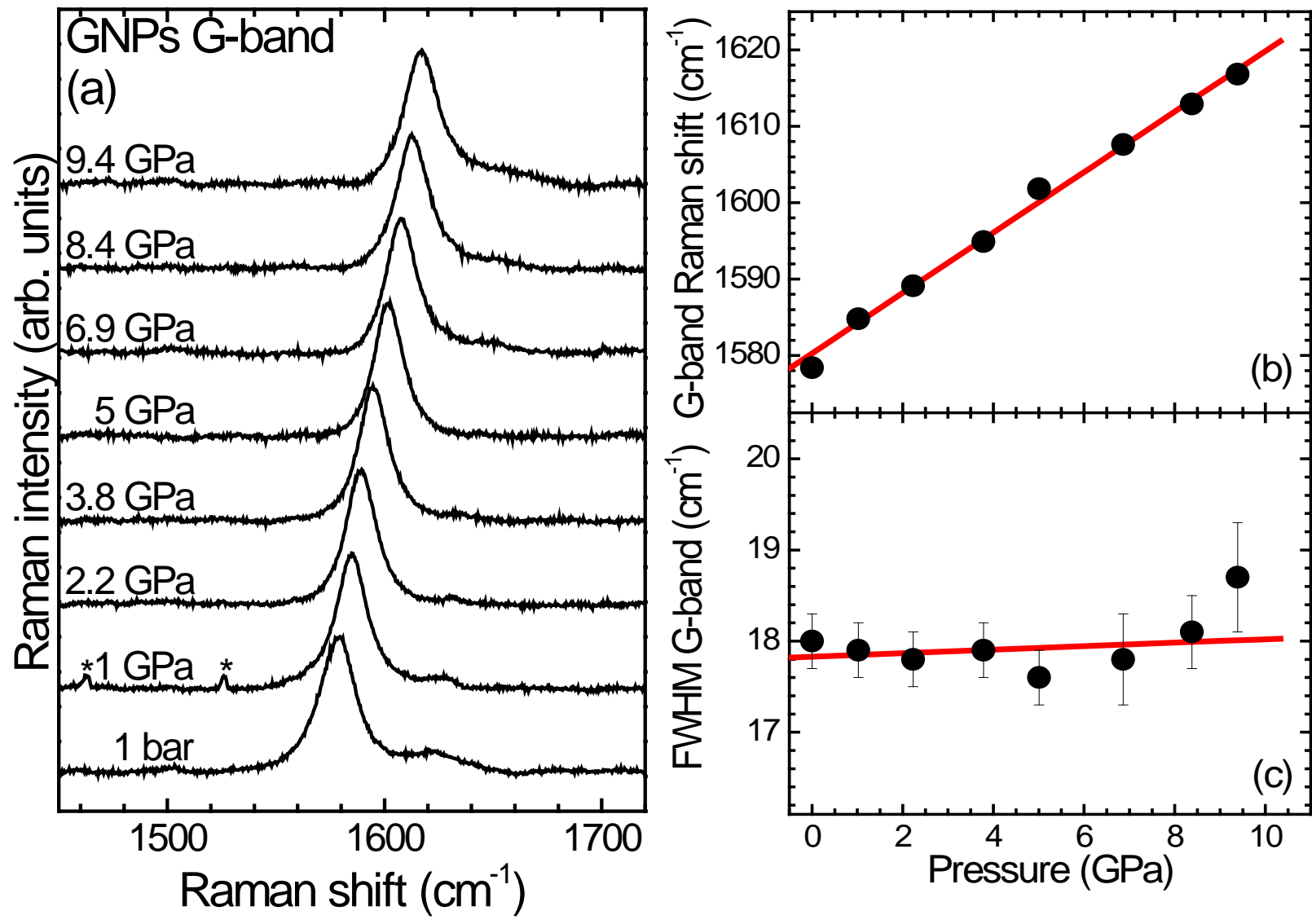

Figure 4. (a) Selected Raman spectra of the G-band of graphene nanoplatelets at various pressures $(\lambda=$ $532 \mathrm{~nm}, T=300 \mathrm{~K})$. Asterisks mark laser plasma lines. The spectra have been normalized with respect to the intense G-band, with the intense DAC diamond-induced Raman background subtracted. Dependence of the (b) Raman mode frequency and (c) full width at half maximum (FWHM) of the G-band against pressure. The spectrometer width contribution of $1 \mathrm{~cm}^{-1}$ has been substracted from the FWHM data. Solid lines passing through the measured data correspond to polynomial fittings. 
We note here that at higher pressures, the G-band undergoes an abrupt pressure-induced broadening (not shown). Interestingly, previous high-pressure Raman experiments on GNPs observed the same effect between 10-15 GPa (depending on the GNPs thickness) ${ }^{36}$ and assigned it to a pressure-induced $\mathrm{sp}^{2}-\mathrm{sp}^{3}$ transition ${ }^{36}$, similar to bulk graphite ${ }^{37-42}$. A more detailed discussion of this effect lies beyond the scope of the present paper.

Turning now to the analysis of the measured Raman data, the Raman mode frequency evolution as a function of pressure can be modelled with the following linear expression:

$$
\omega_{i}^{T}(P)=\omega_{i 0}^{T}+\left(\frac{\partial \omega_{i}}{\partial P}\right)_{T} \Delta P
$$

where $\omega_{\mathrm{i} 0}^{T}$ the mode frequency at ambient conditions, $\Delta P$ the pressure difference, and $\left(\partial \omega_{\mathrm{i}} / \partial P\right)_{\mathrm{T}}$ the first-order pressure coefficient. From our data, we obtain: $\omega_{\mathrm{i} 0}^{\mathrm{T}}=1580.3(1) \mathrm{cm}^{-1}$ and $\left(\partial \omega_{\mathrm{i}} / \partial \mathrm{T}\right)_{T}=3.96(3) \mathrm{cm}^{-1} / \mathrm{GPa}$. We can additionally calculate the respective isothermal mode Grüneisen parameter $\gamma_{i}{ }^{\mathrm{T}}$ :

$$
\gamma_{i}^{T}=\frac{B_{0}}{\omega_{i 0}^{T}}\left(\frac{\partial \omega_{i}}{\partial P}\right)_{T}
$$

where $B_{0}$ the bulk modulus at ambient conditions. Given that by increasing the number of graphene layers composing the (layered) carbon material to more than $\sim 10$, graphene samples tend to 'resemble' graphite in terms of elastic and vibrational properties ${ }^{13,20,31,43-45}$, we can then reasonably use the graphite bulk modulus value $B_{0}=33.8 \mathrm{GPa}^{38}$ in order to get the G-band Grüneisen parameter $\gamma_{\mathrm{i}}^{\mathrm{T}}=0.08(1)$ (the GNPs studied here are composed of ca. 12 layers on average). We should note here that due to the anisotropic nature of graphite-based materials in terms of compressibility, with the $c$-axis being almost one order of magnitude more compressible than the $a$-axis ${ }^{38,40,46-49}$, it is preferable to define Grüneisen parameters for the in-plane and outof-plane vibrational modes separately in these systems ${ }^{38,50,51}$. In the case of the in-plane E2g Gband, the respective Grüneisen parameter $\gamma_{\mathrm{G}}$ can be derived from ${ }^{38,52}$ :

$$
\frac{\omega_{i}^{T}(P)}{\omega_{i 0}^{T}}=\left[\frac{r^{T}(P)}{r_{0}^{T}}\right]^{-3 \gamma_{G}}
$$

where $r^{T}(P)$ and $r_{0}{ }^{T}$ are the hexagonal $a$-axis values at varying and ambient pressure (and ambient temperature), respectively. Assuming the graphite $a$-axis values within the 0-10 GPa pressure range ${ }^{38}$, we calculate $\gamma_{G}=1.05$, in very good agreement with the respective G-band Grüneisen 
parameters for graphite $38,39,53$. Such agreement is to be expected, nevertheless, as high-pressure investigations on graphene samples composed of three layers and more (our GNPs are composed of 12 layers on average) have shown that the pressure-induced G-band frequency shifts are identical to that of graphite ${ }^{51}$. On the other hand, the respective G-band pressure slopes and $\gamma_{G}$ values of (single- or double-layered) graphene depend heavily on the choice of the underlying substrate supporting the samples ${ }^{54,55}$. Since high-pressure measurements on GNPs and graphite do not necessitate the use of substrates, a direct comparison between the vibrational properties of graphene, GNPs, and bulk grahite under compression should be performed with caution.

Having acquired this information at hand from the temperature- and pressure-dependent Raman spectroscopic experments, we can now evaluate the mode-specific phonon anharmonicity of the GNPs’ G-band.

\section{Modeling of the G-band anharmonicity in GNPs}

The shift of a Raman-active mode with varying temperature is generally attributed to thermal expansion (volumetric) and anharmonic effects, and can be approximated by the following equation $^{4,18}$ :

$$
\Delta \omega(T)=\omega_{i}(T)-\omega_{0}=\Delta \omega_{E}(T)+\Delta \omega_{A}(T)
$$

where $\omega_{0}$ represents the harmonic mode frequency at $0 \mathrm{~K}$ (here we use the extrapolated value $\omega_{0}$ $=\omega_{\mathrm{i} 0}^{P}=1581.4(2) \mathrm{cm}^{-1}$ from eq. 1), the $\Delta \omega_{\mathrm{E}}(T)$ component stands for the volumetric thermal expansion contribution (implicit term), and the $\Delta \omega_{\mathrm{A}}(T)$ term describes the anharmonic phononphonon interactions (explicit term) to the mode-specific temperature dependence. The $\Delta \omega_{\mathrm{E}}(T)$ component for the G-band equals to ${ }^{6,18,19,22,23}$ :

$$
\Delta \omega_{E}(T)=\omega_{0}\left[\exp \left(-n \int_{0}^{T} \gamma_{G} \alpha_{\alpha}\left(T^{\prime}\right) d T^{\prime}\right)-1\right]
$$

where $n$ denotes the degeneracy of the Raman-active vibration ( $n=2$ for the G-band with $E_{2 g}$ mode symmetry examined here) and $\alpha_{\alpha}(T)$ the linear thermal expansion coefficient along the inplane hexagonal $a$-axis. Assuming that (a) the isothermal Grüneisen parameter $\gamma_{\mathrm{G}}$ is temperature independent and (b) the thermal expansion coefficient $\alpha_{a}(T)$ of GNPs is identical to that of bulk 
graphite ${ }^{56-60}$, we extract the volumetric /implicit part (CTE) of the G-band frequency shift with temperature [cyan line in Fig. 5(a)].

On the other hand, the anharmonic $\Delta \omega_{\mathrm{A}}(T)$ term can be expressed by the following equation ${ }^{18}$ :

$$
\Delta \omega_{A}(T)=A\left[1+\frac{2}{e^{x}-1}\right]+B\left[1+\frac{3}{e^{y-1}}+\frac{3}{\left(e^{y}-1\right)^{2}}\right]
$$

with $x=\hbar \omega_{0} / 2 \mathrm{k}_{\mathrm{B}} T, y=\hbar \omega_{0} / 3 \mathrm{k}_{\mathrm{B}} T$, $\hbar$ the reduced Planck's constant, kв Boltzmann's constant, and the $A$ and $B$ stand for anharmonic constants acting as fitting parameters. The magnitudes of the $A$ and $B$ constants indicate the strength of the three-(cubic) and four-phonon (quartic) processes, respectively, i.e. the decay of one optical $\Gamma$ phonon into two or three phonons at different points of the Brillouin zone, by obeying energy and momentum conservation ${ }^{61}$. The respective fitted three-phonon (3-ph.) and four-phonon (4-ph.) anharmonic contributions to the GNPs' G-band frequency shift with temperature are shown in Fig. 5(a). We can readily observe that both the CTE and 3-ph. contributions exhibit positive values with increasing temperature [Fig. 5(a)], in excellent agreement with previous observations for graphite samples ${ }^{1,21,23}$. On the contrary, the 4ph. term decreases with increasing temperature, and it becomes obvious that is the dominant mechanism in dictating the temperature dependence of the G-band frequency shift in GNPs, similar to bulk graphite ${ }^{1,21,23}$ and graphene $e^{1,20}$. The fitted parameters are listed in Table 2 alongside relevant literature values.

Comparison with the available literature data reveals some interesting traits. In particular, the values of the $A$ and $B$ parameters for the cubic and quartic phonon decay processes in GNPs are comparable (Table 2), whereas the strength of the cubic decay term is almost two orders of magnitude larger than the respective quartic decay channel in graphite and graphene $e^{1,21,53}$. The exact reasons cannot be identified at this stage, and warrant further investigation. In addition, the $A$ and $B$ parameters in our case exhibit opposite signs, implying that the 3-phonon and 4-phonon processes in GNPs contributing to the temperature dependence of the G-band frequency shift involve different conversion procedures ${ }^{62}$. More precisely, the positive $A$ value of the 3 -phonon process (Table 2) can be translated into an asymmetric up-conversion channel, where the $\mathrm{E}_{2 \mathrm{~g}} / \mathrm{G}$ band mode at the center of the Brillouin zone ( $\Gamma$-point) will decay into a higher frequency (optical) and a lower frequency (acoustical) phonon with opposite momenta. Considering the 
maxima of the graphite and graphene phonon density of states (PDOS) ${ }^{1,21,63}$, one such possible up-conversion channel is: $\mathrm{E}_{2 \mathrm{~g}}\left(\Gamma, 1581 \mathrm{~cm}^{-1}\right) \rightarrow \mathrm{LO}\left(\Gamma-\mathrm{K}, 1600 \mathrm{~cm}^{-1}\right)-\mathrm{ZA}\left(\Gamma-\mathrm{K}, 20 \mathrm{~cm}^{-1}\right)$.

In a similar fashion, the negative $B$ value for the 4-phonon quartic process (Table 2) must be related mainly to down-conversion decay channels, i.e. the decay of the G-band into three lower energy phonons such as: $\mathrm{E}_{2 \mathrm{~g}}\left(\Gamma, 1581 \mathrm{~cm}^{-1}\right) \rightarrow \mathrm{ZO}\left(\Gamma, 800 \mathrm{~cm}^{-1}\right)+\mathrm{ZA}\left(\Gamma-\mathrm{K}, 400 \mathrm{~cm}^{-1}\right)+\mathrm{ZA}(\Gamma-$ $\left.\mathrm{K}, 400 \mathrm{~cm}^{-1}\right)^{1,21,63}$. A more detailed discussion, however, would require an accurate measurement of the GNPs' PDOS in combination with $a b$ initio calculations, extending beyond the scope of the present paper.

Table 2. Fitting parameters derived from reproducing the Raman-active G-band frequency and width shift as a function of temperature in GNPs, according to eqs. 6-11. Literature data are also listed for comparison. N/A: Not Available.

\begin{tabular}{c|cccccccc}
\hline \hline $\begin{array}{c}\text { Carbon samples } \\
\text { (pristine) }\end{array}$ & $\begin{array}{c}\omega_{0} \\
\left(\mathrm{~cm}^{-1}\right)\end{array}$ & $\begin{array}{c}A \\
\left(\mathrm{~cm}^{-1}\right)\end{array}$ & $\begin{array}{c}B \\
\left(\mathrm{~cm}^{-1}\right)\end{array}$ & $\begin{array}{c}\Gamma(0) \\
\left(\mathrm{cm}^{-1}\right)\end{array}$ & $\begin{array}{c}\Gamma_{0} \\
\left(\mathrm{~cm}^{-1}\right)\end{array}$ & $\begin{array}{c}\Gamma_{\text {EPC }} \\
(0) \\
\left(\mathrm{cm}^{-1}\right)\end{array}$ & $\begin{array}{c}C \\
\left(\mathrm{~cm}^{-1}\right)\end{array}$ & $\begin{array}{c}D \\
\left(\mathrm{~cm}^{-1}\right)\end{array}$ \\
\hline GNPs & $1581.4(2)$ & $8.3(3)$ & $-9.3(1)$ & 19 & $2.1^{\mathbf{a}}$ & 12 & 3.1 & 1.8 \\
Graphite $^{23}$ & 1583.5 & N/A & N/A & 11.88 & $0^{\mathbf{a}}$ & 10.3 & 0.84 & 0.74 \\
Graphite $^{53}$ & 1602 & -19.37 & -0.237 & 15.84 & $3.84^{\mathbf{b}}$ & 9.8 & 1.31 & 0.89 \\
Graphite $^{1,21}$ & 1583 & -14.1 & 1.3 & $11.5^{\mathbf{a}}$ & N/A & 11.01 & N/A & N/A \\
${\text { Graphene (single-layer })^{1}}^{1}$ & N/A & -14.02 & 3.03 & N/A & N/A & N/A & N/A & N/A \\
Graphene sheets $^{22}$ & 1582 & -1 & -6 & $\sim 17.5^{\mathbf{a}}$ & N/A & 9 & 7 & 1.5 \\
\hline \hline
\end{tabular}

${ }^{\mathbf{a}}$ The width contribution due to the finite resolution of the spectrometer has been substracted.

b Attributed to the finite resolution of the spectrometer.

We turn now to the width $\Gamma$ of the G-band as a function of temperature. Generally, the broadening of the G-band (or lifetime of the respective phonon) can be accounted for by two physical processes $^{1,19}$ :

$$
\Gamma(T)=\Gamma_{0}+\Gamma^{E P C}(T)+\Gamma^{a n}(T)
$$

where the temperature-independent $\Gamma_{0}$ term stands for the width contribution due to the finite spectrometer resolution and/or other scattering mechanisms such as crystalline defects and impurities present in real samples ${ }^{64}$, and $\Gamma^{\mathrm{EPC}}$ and $\Gamma^{\text {an }}$ denoting the contributions from the electron-phonon coupling (EPC, decay of the phonon into a hole-electron pair) and the 
anharmonic phonon-phonon interactions (phonon-phonon scattering), respectively. The $\Gamma^{\mathrm{EPC}}$ term for the graphite- and graphene systems can be approximated by ${ }^{1}$ :

$$
\Gamma^{E P C}(T)=\Gamma^{E P C}(0)\left[\frac{1}{e^{-\frac{\hbar \omega_{0}}{2 k_{B} T}+1}}-\frac{1}{e^{\frac{\hbar \omega_{0}}{2 k_{B} T}}+1}\right]
$$

whereas the anharmonic $\Gamma^{\text {an }}$ part can be expressed as ${ }^{1,4,18,19}$ :

$$
\Gamma^{a n}(T)=C\left[1+\frac{2}{e^{x}-1}\right]+D\left[1+\frac{3}{e^{y-1}}+\frac{3}{\left(e^{y-1}\right)^{2}}\right]
$$

with $x=\hbar \omega_{0} / 2 \mathrm{k}_{\mathrm{B}} T, y=\hbar \omega_{0} / 3 \mathrm{k}_{\mathrm{B}} T$, and the $C$ and $D$ stand acting as fitting parameters for the three- and four-phonon decay processes. The respective fittings are shown in Fig. 5(b), with the calculated parameters listed in Table 2.

We can readily observe that the most important contribution in the G-band width comes from the EPC term, consistent with previous observations in similar carbon systems ${ }^{1,20-23}$. We should mention here that the latter decreases with increasing temperature, since this EPC term can be considered proportional to the difference between the occupation of the electronic states below and above the Fermi energy $\mathrm{EF}_{\mathrm{F}}$; as temperature increases, the occupation of filled states below EF decreases, while the empty states above EF become more occupied, resulting in the observed decrease of the EPC factor with temperature ${ }^{1,2}$. On the other hand, the anharmonic phononphonon scattering term $\Gamma^{\mathrm{an}}$ will always increase with increasing temperature ${ }^{65,66}$, with the cubic 3ph. and the quartic 4-ph. terms exhibiting linear $(T)$ and $T^{2}$ temperature dependences, respectively. Contrary to pristine graphite, however, the width of the GNPs' G-band exhibits a (moderate) increase with increasing temperature, thus resembling more the behavior of graphene in that aspect ${ }^{20,22}$. Consequently, this temperature-induced broadening of the G-band denotes the underlying importance of the anharmonic phonon-phonon interactions in determining the lifetime, as well as the frequency of the in-plane $\mathrm{E}_{2 \mathrm{~g}}$ carbon-stretching vibration in these systems. Finally, we should point out that the finite value of $\Gamma_{0}=2.1 \mathrm{~cm}^{-1}$ found in the current work (Table 2) corresponds to scattering processes due to the presence of the Fe- and Cr-rich impurities present $n$ the investigated GNPs (Figs. 1 \& 2). 


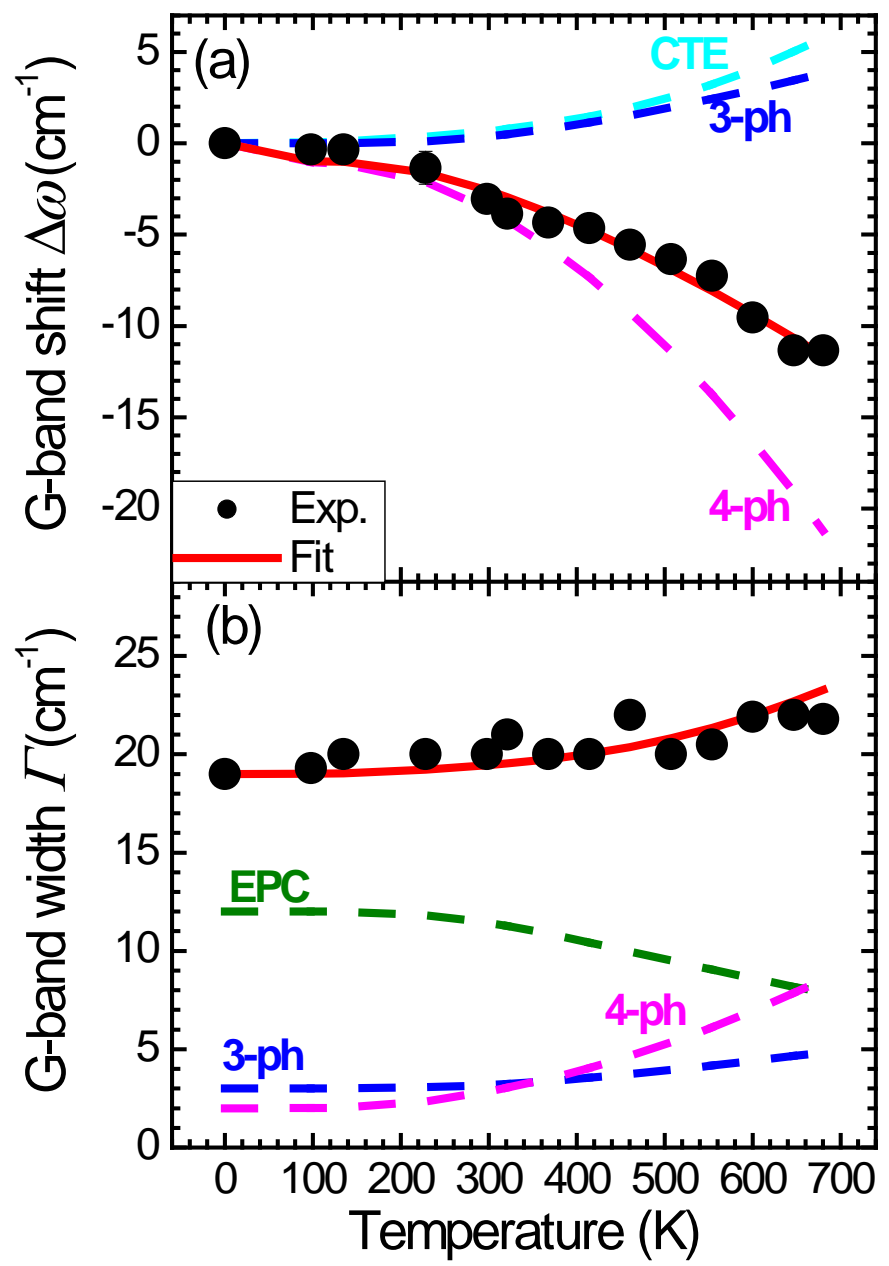

Figure 5. (a) Frequency shift and (b) width of the GNPs' G-band as a function of temperature. The experimental data are drawn as solid symbols, and the red solid lines correspond to total fittings according to eqs. 6-11. The various contributions are drawn separately as dashed lines. The fitting parameters are listed in Table 2.

\section{CONCLUSIONS}

In summary, we have investigated the effect of temperature and pressure on the Raman-active G-band of graphene nanoplatelets. Measuring the temperature- and pressure-induced Raman mode frequency and width shift allowed us to extract the individual contributions to the G-band, i.e. the volumetric (thermal expansion) and anharmonic (phonon-phonon interactions) terms responsible for the Raman shift with temperature, and the electron-phonon coupling and anharmonic contributions controlling the width/lifetime of the G-band as a function of 
temperature. Comparison with relevant systems indicates the significant role of the anharmonic effects for understanding the G-band physical processes in carbon-based systems.

\section{Acknowledgements}

We would like to thank Dr. S. Tkachev at GeoSoilEnviroCARS (Sector 13), APS-ANL for his assistance with the DAC gas loading. Use of the COMPRES-GSECARS gas loading system was supported by COMPRES under NSF Cooperative Agreement EAR -1606856 and by GSECARS through NSF grant EAR-1634415 and DOE grant DE-FG02-94ER14466. This research used resources of the Advanced Photon Source, a U.S. Department of Energy (DOE) Office of Science User Facility operated for the DOE Office of Science by Argonne National Laboratory under Contract No. DE-AC02-06CH11357. A. T. was supported by the Summer Materials Reseach Training (SMaRT) program, funded by the National Science Foundation. The financial support of the Helmholtz Recruiting Initiative (grant No. I-044-16-01) is acknowledged. Portion of this research was supported by the Faculty Research Grant of Oakland University, Michigan, U.S.A.

\section{References}

(1) Bonini, N.; Lazzeri, M.; Marzari, N.; Mauri, F. Phonon Anharmonicities in Graphite and Graphene. Phys. Rev. Lett. 2007, 99, 176802.

(2) Bonini, N.; Rao, R.; Rao, A. M.; Marzari, N.; Menendez, J. Lattice Anharmonicity in LowDimensional Carbon Systems. Phys. Stat. Sol. 2008, 245, 2149-2154.

(3) Calizo, I.; Balandin, A. A.; Bao, W.; Miao, F.; Lau, C. N. Temperature Dependence of the Raman Spectra of Graphene and Graphene Multilayers. Nano Lett. 2007, 7, 2645-2649.

(4) Lucazeau, G. Effect of Pressure and Temperature on Raman Spectra of Solids: Anharmonicity. J. Raman Spectr. 2003, 34, 478-496.

(5) Mishra, K. K.; Ghosh, S.; Ravindran, T. R.; Amirthapandian, S.; Kamruddin, M. Thermal Conductivity and Pressure-Dependent Raman Studies of Vertical Graphene Nanosheets. J. Phys. Chem. C 2016, 120, 25092-25100.

(6) Postmus, C.; Ferraro, J. R.; Mitra, S. S. Pressure Dependence of Infrared Eigenfrequencies of KCl and KBr. Phys. Rev. 1968, 174, 983-987.

(7) Cai, W.; Moore, A. L.; Zhu, Y.; Li, X.; Chen, S.; Shi, L.; Ruoff, R. S. Thermal Transport in Suspended and Supported Monolayer Graphene Grown by Chemical Vapor Deposition. Nano Lett. 2010, 10, 1645-1651.

(8) Balandin, A. A.; Ghosh, S.; Bao, W.; Calizo, I.; Teweldebrhan, D.; Miao, F.; Lau, C. N. Superior Thermal Conductivity of Single-Layer Graphene. Nano Lett. 2008, 3, 902-907. 
(9) Ghosh, S.; Calizo, I.; Teweldebrhan, D.; Pokatilov, E. P.; Nika, D. L.; Balandin, A. A.; Bao, W.; Miao, F.; Lau, C. N. Extremely High Thermal Conductivity of Graphene: Prospects for Thermal Management Applications in Nanoelectronic Circuits. Appl. Phys. Lett. 2008, 92, 151911.

(10) Faugeras, C.; Faugeras, B.; Orlita, M.; Potemski, M.; Nair, R. R.; Geim, A. K. Thermal Conductivity of Graphene in Corbino Membrane Geometry. ACS Nano 2010, 4, 1889-1892.

(11) Li, Q.-Y.; Xia, K.; Zhang, J.; Zhang, Y.; Li, Q.; Takahashi, K.; Zhang, X. Measurement of Specific Heat and Thermal Conductivity of Supported and Suspended Graphene by a Comprehensive Raman Optothermal Method. Nanoscale 2017, 9, 10784-10793.

(12) Malekpour, H.; Balandin, A. A. Raman-Based Technique for Measuring Thermal Conductivity of Graphene and Related Materials. J. Raman Spectr. 2018, 49, 106-120.

(13) Ferrari, A. C.; Basko, D. M. Raman Spectroscopy as a Versatile Tool for Studying the Properties of Graphene. Nat. Nanotechnol. 2013, 8, 235-246.

(14) Shahil, K. M. F.; Balandin, A. A. Thermal Properties of Graphene and Multilayer Graphene: Applications in Thermal Interface Materials. Sol. St. Comm. 2012, 152, 1331-1340.

(15) Late, D. J.; Maitra, U.; Panchakarla, L. S.; Waghmare, U. V; Rao, C. N. R. Temperature Effects on the Raman Spectra of Graphenes: Dependence on the Number of Layers and Doping. J. Phys. Cond. Matt. 2011, 23, 55303.

(16) Malard, L. M.; Moreira, R. L.; Elias, D. C.; Plentz, F.; Alves, E. S.; Pimenta, M. A. Thermal Enhancement of Chemical Doping in Graphene: A Raman Spectroscopy Study. J. Phys. Cond. Matt. 2010, 22, 334202.

(17) Cataldi, P.; Athanassiou, A.; Bayer, I. S. Graphene Nanoplatelets-Based Advanced Materials and Recent Progress in Sustainable Applications. Appl. Sci. 2018, 8, 1438.

(18) Balkanski, M.; Wallis, R. F.; Haro, E. Anharmonic Effects in Light Scattering Due to Optical Phonons in Silicon. Phys. Rev. B 1983, 28, 1928-1934.

(19) Menendez, J.; Cardona, M. Temperature Dependence of the First-Order Raman Scattering by Phonons in Si, Ge, and $\alpha$-Sn: Anharmonic Effects. Phys. Rev. B 1984, 29, 2051-2059.

(20) Apostolov, A. T.; Apostolova, I. N.; Wesselinowa, J. M. Temperature and Layer Number Dependence of the G and 2D Phonon Energy and Damping in Graphene. J. Phys. Cond. Matt. 2012, 24, 235401.

(21) Giura, P.; Bonini, N.; Creff, G.; Brubach, J. B.; Roy, P.; Lazzeri, M. Temperature Evolution of Infrared- and Raman-Active Phonons in Graphite. Phys. Rev. B 2012, 86, 121404(R).

(22) Lin, J.; Guo, L.; Huang, Q.; Jia, Y.; Li, K.; Lai, X.; Chen, X. Anharmonic Phonon Effects in Raman Spectra of Unsupported Vertical Graphene Sheets. Phys. Rev. B 2011, 83, 125430.

(23) Liu, H. N.; Cong, X.; Lin, M. L.; Tan, P. H. The Intrinsic Temperature-Dependent Raman Spectra of Graphite in the Temperature Range 4 K to 1000 K. Carbon N. Y. 2019, 152, 451-458.

(24) Trittschack, R.; Grobety, B.; Koch-Müller, M. In Situ High-Temperature Raman and FTIR Spectroscopy of the Phase Transformation of Lizardite. Amer. Miner. 2012, 97, 1965-1976.

(25) Schück, G.; Többens, D. M.; Koch-Müller, M.; Efthimiopoulos, I.; Schorr, S. Infrared Spectroscopic Study of Vibrational Modes across the Orthorhombic-Tetragonal Phase Transition in 
Methylammonium Lead Halide Single Crystals. J. Phys. Chem. C 2018, 122, 5227-5237.

(26) Syassen, K. Ruby under Pressure. High Press. Res. 2008, 28, 75-126.

(27) Owen, E. A.; Yates, E. L. Precision Measurements of Crystal Parameters. Philos. Mag. 1933, 15, 472-488.

(28) Trucano, P.; Chen, R. Structure of Graphite by Neutron Diffraction. Nature 1975, 258, 136-137.

(29) Tuinstra, F.; Koenig, J. L. Raman Spectrum of Graphite. J. Chem. Phys. 1970, 53, 1126-1130.

(30) Pimenta, M. A.; Dresselhaus, G.; Dresselhaus, M. S.; Cancado, L. G.; Jorio, A.; Saito, R. Studying Disorder in Graphite-Based Systems by Raman Spectroscopy. Phys. Chem. Chem. Phys. 2007, 9, 1276-1291.

(31) Ferrari, A. C. Raman Spectroscopy of Graphene and Graphite: Disorder, Electron-Phonon Coupling, Doping and Nonadiabatic Effects. Sol. St. Comm. 2007, 143, 47-57.

(32) Cancado, L. G.; Takai, K.; Enoki, T.; Endo, M.; Kim, Y. A.; Mizusaki, H.; Jorio, A.; Coelho, L. N.; Magalhaes-Paniago, R.; Pimenta, M. A. General Equation for the Determination of the Crystallite Size $L_{\alpha}$ of Nanographite by Raman Spectroscopy. Appl. Phys. Lett. 2006, 88, 163106.

(33) Tan, P.; Deng, Y.; Zhao, Q.; Cheng, W. The Intrinsic Temperature Effect of the Raman Spectra of Graphite. Appl. Phys. Lett. 1999, 74, 1818-1820.

(34) Pawbake, A. S.; Mishra, K. K.; Machuno, L. G. B.; Gelamo, R. V; Ravindran, T. R.; Rout, C. S.; Late, D. J. Temperature and Pressure Dependent Raman Spectroscopy of Plasma Treated Multilayer Graphene Nanosheets. Diam. Relat. Mater. 2018, 84, 146-156.

(35) Zouboulis, E. S.; Grimsditch, M. Raman Scattering in Diamond up to 1900 K. Phys. Rev. B 1991, 43, 12490-12493.

(36) Lu, S.; Yao, M.; Yang, X.; Li, Q.; Xiao, J.; Yao, Z.; Jiang, L.; Liu, R.; Liu, B.; Chen, S.; et al. High Pressure Transformation of Graphene Nanoplates: A Raman Study. Chem. Phys. Lett. 2013, 585, 101.

(37) Amsler, M.; Flores-Livas, J. A.; Lehtovaara, L.; Balima, F.; Ghasemi, S. A.; Machon, D.; Pailhes, S.; Willand, A.; Caliste, D.; Botti, S.; et al. Crystal Structure of Cold Compressed Graphite. Phys. Rev. Lett. 2012, 108, 65501.

(38) Hanfland, M.; Beister, H.; Syassen, K. Graphite under Pressure: Equation of State and First-Order Raman Modes. Phys. Rev. B 1989, 39, 12598.

(39) Goncharov, A. F. Graphite at High Pressures: Amorphization at 44 GPa. High Press. Res. 1992, 8, 607-616.

(40) Wang, Y.; Panzik, J. E.; Kiefer, B.; Lee, K. K. M. Crystal Structure of Graphite under RoomTemperature Compression and Decompression. Sci. Reports 2012, 2, 520.

(41) Wang, Y.; Lee, K. K. M. From Soft to Superhard: Fifty Years of Experiments on ColdCompressed Graphite. J. Superhard Mater. 2012, 34, 360-370.

(42) Mao, W. L.; Mao, H.-K.; Eng, P. J.; Trainor, T. P.; Newville, M.; Kao, C.-C.; Heinz, D. L.; Shu, J.; Meng, Y.; Hemley, R. J. Bonding Changes in Compressed Superhard Graphite. Science. 2003, 302, 425. 
(43) Graf, D.; Molitor, F.; Ensslin, K.; Stampfer, C.; Jungen, A.; Hierold, C.; Wirtz, L. Spatially Resolved Raman Spectroscopy of Single- and Few-Layer Graphene. Nano Lett. 2007, 7, 238-242.

(44) Michel, K. H.; Verbeck, B. Theory of the Evolution of Phonon Spectra and Elastic Constants from Graphene to Graphite. Phys. Rev. B 2008, 78, 85424.

(45) Tan, P. H.; Han, W. P.; Zhao, W. J.; Wu, Z. H.; Chang, K.; Wang, H.; Wang, Y. F.; Bonini, N.; Marzari, N.; Pugno, N.; et al. The Shear Mode of Multilayer Graphene. Nat. Mater. 2012, 11, 294300.

(46) Clark, S. M.; Jeon, K.-J.; Chen, J.-Y.; Yoo, C.-S. Few-Layer Graphene under High Pressure: Raman and X-Ray Diffraction Studies. Sol. St. Comm. 2013, 154, 15-18.

(47) Martins, L. G. P.; Silva, D. L.; Smith, J. S.; Lu, A.-Y.; Su, C.; Hempel, M.; Occhialini, C.; Ji, X.; Pablo, R.; Alencar, R. S.; et al. Evidence for a Pressure-Induced Phase Transition of Few-Layer Graphene to 2D Diamond. arXiv: 1910.015912019.

(48) Yagi, T.; Utsumi, W.; Yakamata, M.; Kikegawa, T.; Shimomura, O. High-Pressure in Situ X-Ray Diffraction Study of the Phase Transformation from Graphite to Hexagonal Diamond at Room Temperature. Phys. Rev. B 1992, 46, 6031-6039.

(49) Zhao, Y. X.; Spain, I. L. X-Ray Diffraction Data for Graphite to 20 GPa. Phys. Rev. B 1989, 40, 993-997.

(50) Mohiuddin, T. M. G.; Lombardo, A.; Nair, R. R.; Bonetti, A.; Savini, G.; Jalil, R.; Bonini, N.; Basko, D. M.; Galiotis, C.; Marzari, N.; et al. Uniaxial Strain in Graphene by Raman Spectroscopy: G Peak Splitting, Grüneisen Parameters, and Sample Orientation. Phys. Rev. B 2009, 79, 205433.

(51) Proctor, J. E.; Gregoryanz, E.; amd M. Lotya, K. S. N.; Coleman, J. N.; Halsall, M. P. HighPressure Raman Spectroscopy of Graphene. Phys. Rev. B 2009, 80, 73408.

(52) Zallen, R. Pressure-Raman Effects and Vibrational Scaling Laws in Molecular Crystals: $\mathrm{S}_{8}$ and $\mathrm{As}_{2} \mathrm{~S}_{3}$. Phys. Rev. B 1974, 9, 4485-4496.

(53) Montagnac, G.; Caracas, R.; Bobocioiu, E.; Vittoz, F.; Reynard, B. Anharmonicity of Graphite from UV Raman Spectroscopy to 2700 K. Carbon N. Y. 2013, 54, 68-75.

(54) Machon, D.; Bousige, C.; Alencar, R.; Torres-Dias, A.; Balima, F.; Nicolle, J.; Pinheiro, G. S.; Filho, A. G. S.; Miguel, A. S. Raman Scattering Studies of Graphene under High Pressure. J. Raman Spectr. 2018, 49, 121-129.

(55) Filintoglou, K.; Papadopoulos, N.; Arvanitidis, J.; Christofilos, D.; Frank, O.; Kalbac, M.; Parthenios, J.; Kalosakas, G.; Galiotis, C.; Papagelis, K. Raman Spectroscopy of Graphene at High Pressure: Effects of the Substrate and the Pressure Transmitting Media. Phys. Rev. B 2013, 88, 45418.

(56) Bailey, A. C.; Yates, B. Anisotropic Thermal Expansion of Pyrolytic Graphite at Low Temperatures. J. Appl. Phys. 1970, 41, 5088-5091.

(57) Tsang, D. K. L.; Marsden, B. J.; Fok, S. L.; Hall, G. Graphite Thermal Expansion Relationship for Different Temperature Ranges. Carbon N. Y. 2005, 43, 2902-2906.

(58) Morgan, W. C. Thermal Expansion Coefficients of Graphite Crystals. Carbon N. Y. 1972, 10, 7379. 
(59) Mounet, N.; Marzari, N. First-Principles Determination of the Structural, Vibrational and Thermodynamic Properties of Diamond, Graphite, and Derivatives. Phys. Rev. B 2005, 71, 205214.

(60) Marsden, B.; Mummery, A.; Mummery, P. Modelling the Coefficient of Thermal Expansion in Graphite Crystals: Implications of Lattice Strain Due to Irradiation and Pressure. Proc. R. Soc. A 2018, 474, 20180075.

(61) Klemens, P. G. Anharmonic Decay of Optical Phonons. Phys. Rev. B 1966, 148, 845-848.

(62) Zhao, Z.; Elwood, J.; Carpenter, M. A. Phonon Anharmonicity of PdO Studied by Raman Spectroscopy. J. Phys. Chem. C 2015, 119, 23094-23102.

(63) Mohr, M.; Maultzsch, J.; Dobardzic, E.; Reich, S.; Milosevic, I.; Damnjanovic, M.; Bosak, A.; Krisch, M.; Thomsen, C. Phonon Dispersion of Graphite by Inelastic X-Ray Scattering. Phys. Rev. B 2007, 76, 35439.

(64) Verma, P.; Abbi, S. C.; Jain, K. P. Raman-Scattering Probe of Anharmonic Effects in GaAs. Phys. Rev. B 1995, 51, 16660-16667.

(65) Ipatova, I. P.; Maradudin, A. A.; Wallis, R. F. Temperature Dependence of the Width of the Fundamental Lattice-Vibration Absorption Peak in Ionic Crystals. II. Approximate Numerical Results. Phys. Rev. 1967, 155, 882-895.

(66) Monga, M. R.; Jindal, V. K.; Pathak, K. N. Self-Energy of Phonons in an Anharmonic Crystal of Order L4. III. Approximate Numerical Results for Ionic Crystals. Phys. Rev. B 1979, 19, 12301242. 


\section{TOC Graphic}
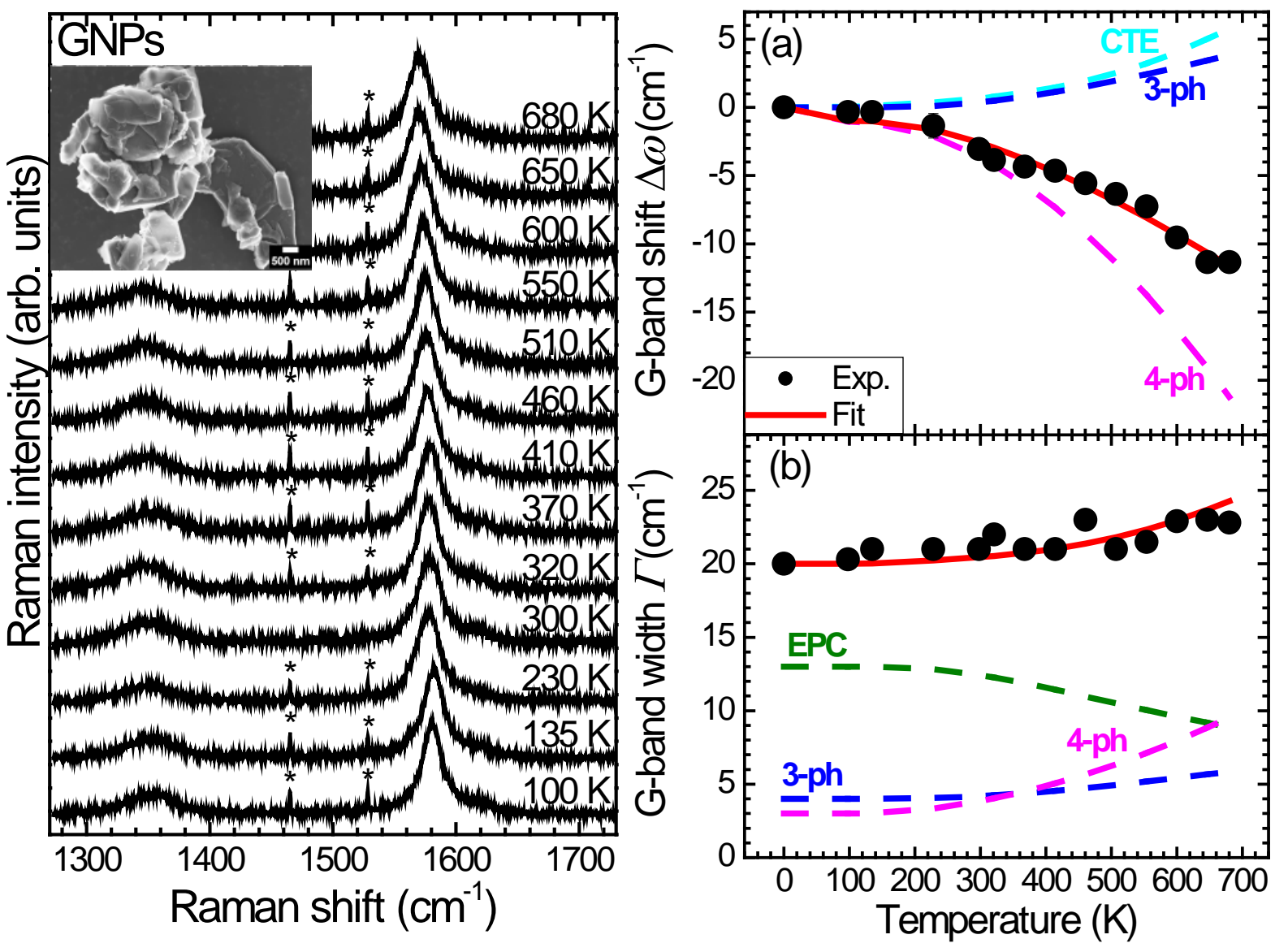